\title{
Diseño y construcción de un dedo para
}

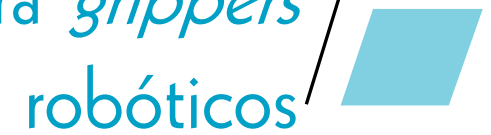

Óscarf. Aviles*

Pedro L. Simanca**

Paola A. Niño***

\section{Resumen}

En este trabajo muestra el diseño de un prototipo de dedo robótico antropomórfico ${ }^{l,}$ un dedo artificial para la posterior implementación de una mano bio-mecatrónica. El dedo es desarrollado en el Laboratorio de Automatización y Robótica de la Universidad Estatal de Campinas (Brasil) con la colaboración de la Universidad Militar Nueva Granada en Bogotá(Colombia). Una mano robótica multidedos típicamente tiene muchos grados de libertad (GDL), estos dedos están montados en una palma rígida, la cual está proyectada para ser ensamblada en la muñeca de un brazo robótico. El principal componente de una mano multidedos es el mecanismo de un dedo. Con base en el estudio de manos robóticas disponibles, es desarrollado un mecanismo para un dedo artificial el cual posee tres juntas y un GDL para todo el mecanismo. El mecanismo implementado es un sistema de palancas donde la entrada es un tornillo sin fin para conversión de movimiento rotacional en lineal. Un prototipo del dedo es implementado y probado experimentalmente. Este diseño presenta una alternativa de bajo costo y permite la actuación y control de una mano artificial con un número pequeño de grados de libertad.

Palabras clave: antropomorfo, anatomía, simulación, robótica.

\section{Design and construction of a finger for robotic}

\begin{abstract}
This work presents the design of a prototype of an anthropomorphic robotic finger (12). The main objective of this article is to show the design of an artificial finger. It describes the implementation of a fingerfor the implementation of a Bio-Mecatronic hand. The finger is developed in the "Laboratorio de Automatización y Robótica de la Universidad Estatal de Campinas (Brazil)" with collaboration with the "Universidad Militar Nueva Granada, Bogotá", (Colombia). A robotic hand multi fingers has many grades of liberty (GDL), these fingers are placed on a rigid palm, projected to be assembled in the

* Ingeniero eléctrico, MSc. Estudiante de doctorado, Universidad de Campinas (Brasil). oaviles@ fem.unicamp.br

** Ingeniero eléctrico, MSc. PhD. Universidad Militar Nueva Granada

** Ingeniero mecánico, MSc
\end{abstract}


wrist of a robotic arm. The main component of a multi finger hand is the mechanism of one finger. Based on the study of robotic hands available, a mechanism is developed for an artificial finger with three joints and one GDL for all the mechanism. The implemented mechanism is a system of levers where the entrance is a worm screw for the conversion of rotational movement into lineal. A prototype of a finger is implemented and tested experimentally. This design presents an alternative of low cost and allows the action and control of an artificial hand with some liberty.

Key Words: anthropomorphic, anatomy, simulation, robotics.

\section{Introducción}

El agarre humano posee una sorprendente flexibilidad e incomparable destreza, tal que ha llamado la atención de muchos investigadores. El estudio del agarre humano como modelo ha llevado a la construcción de innovadores pero costosos prototipos de dedos, para ser utilizados como partes funcionales de robots antropomórficos. Ejemplos de referencia son los dedos utilizados en las manos de Stanford/JPL hand, Utah/MIT hand, TUAT/Karlsruhe Humanoid Hand, DLR Hand, Robonaut hand de la Nasa[6], Mano ultraleve, UMNG Hand. Los cuales se pueden observar en la figura 1 .

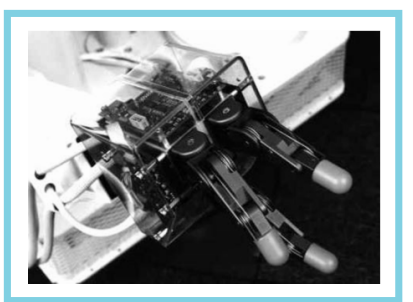

Stanford/JPL [1]

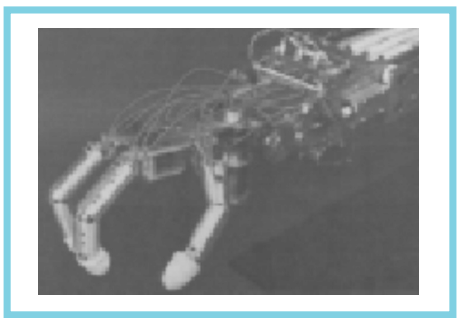

Hitachi [3]

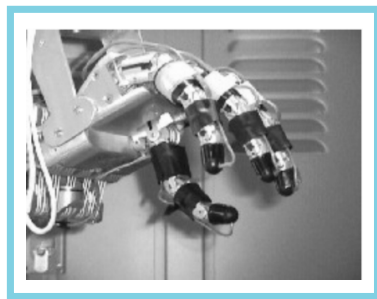

Utah / MIT [2]

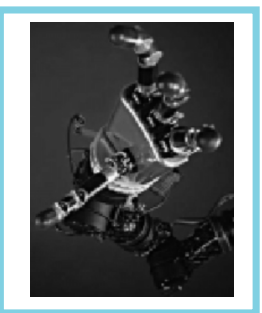

DLR [4]

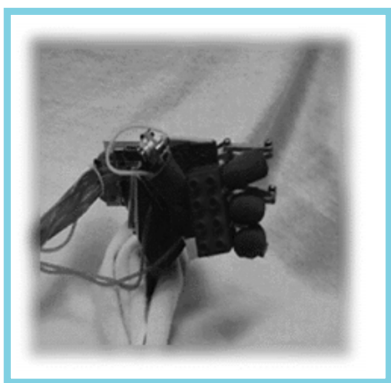

Mano de Vanderbilt [12].

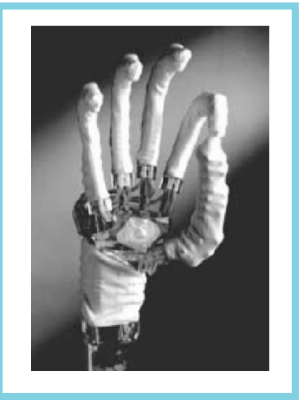

Mano Ultraleve [7]

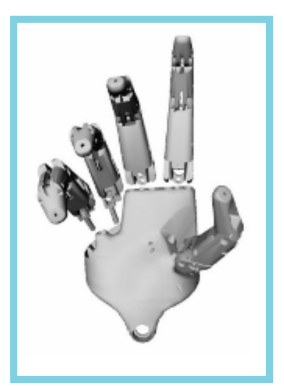

Robonaut da Nasa [6]

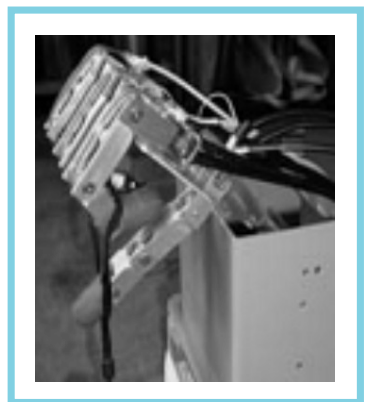

Mano UMNG [12]
Figura 1. Ejemplos de manos desarrolladas por centros de investigación a nivel mundial.

La mayoría de los prototipos disponibles tienen un número alto de GDL, un control complejo y por tanto un alto costo. Recientemente se han desarrollado estudios en la Universidad Militar Nueva Granada en el laboratorio de Robótica e Inteligencia artificial (LRIA) en donde se desarrolló una mano antropomórfica que imita los modelos prensiles humanos pero cuyo inconveniente sigue siendo el elevado número de actuadores utilizados y el costo para su implementación.

En este artículo se trata el diseño de prototipo de dedo antropomórfico, pues tiene el tamaño si- 
milar al de un dedo índice humano y utiliza un menor número de actuadores, además de ser económico y de funcionamiento sencillo. Este prototipo puede usarse como módulo para una mano robótica antropomorfa. Para el diseño de este mecanismo ha sido de gran importancia el uso de software de simulación mecánica como SolidWorks® y Visual Nastran ${ }^{\circledR}$, con el fin de tomar decisiones de los elementos que se deben usar para la trasmisión de movimiento entre el actuador (motor CC) y las partes móviles que componen la estructura.

\section{La mano humana}

El diseño de órganos de agarre tiene como fuente natural de inspiración la mano humana. El objetivo de los investigadores es la emulación de las habilidades mostradas por la mano en su interacción con el ambiente externo.

La mano humana se conecta a la muñeca a través de la palma y está dotada de veinte GDL accionados por cerca de cuarenta músculos. La estructura ósea de la mano se muestra en la figura. 2

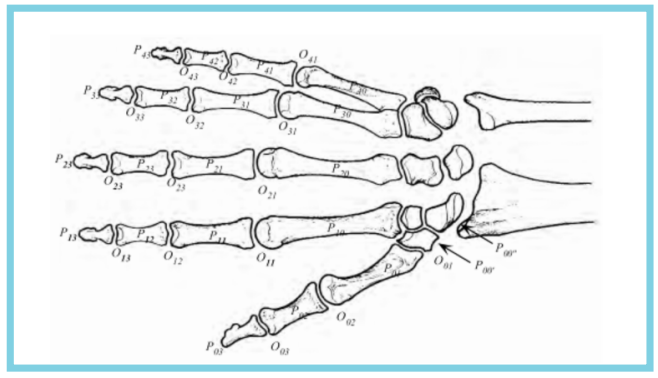

a) Esqueleto de la mano humana y sistemas de referencia asociados.

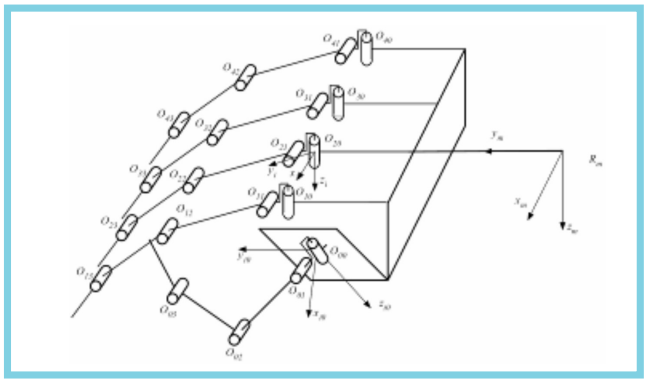

b) Modelo mecánico equivalente.

Figura 2. Esqueleto de la mano y modelo mecánico equivalente.
Como se observa, el dedo pulgar está fijo por debajo de los otros dedos y puede realizar los movimientos de cierre y rotación, debido a la gran movilidad de su metacarpo. Esto permite variar la orientación del plano en que se desarrolla el movimiento de doblado y extensión del dedo pulgar, propiedad a través de la cual es posible oponer el dedo pulgar a los otros dedos. Con el término abducción se entiende el movimiento de salida del dedo del eje del brazo.

El movimiento de extensión/abducción es la capacidad de extensión del pulgar hacia la parte exterior y flexión hacia el interior de la palma. El término Oposición se define como la capacidad de unión de las puntas del pulgar y el meñique.

La aducción/abducción es la capacidad de acercamiento y alejamiento del pulgar de la palma, cuando ambos se encuentran en un mismo plano.

La gran cantidad de músculos y juntas que están presentes en la mano permiten esta gran variedad de configuraciones de agarre. En 1919, Schlesinger desarrolló una clasificación de la taxonomía para el estudio de la destreza de las manos humanas. Este autor agrupó en seis categorías las estrategias de agarre de la mano humana: agarre cilíndrico, de punta, de gancho, de palma (Palmar), esférico y de lado (Lateral), como es mostrado en la figura.

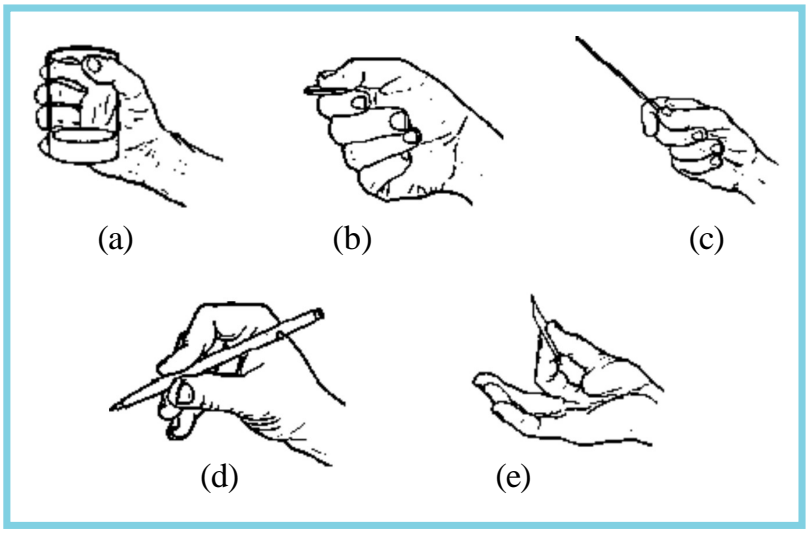

Figura 3. modelos prensiles humanos, a) cilíndrica palmar, b) subtérmino lateral, c) centralizado, d) tridigital, e) posición subterminal. 


\section{Diseño dedo articulado}

Con base en estudios de la biomecánica de la mano y de sus medidas antropométricas, ver tabla 1, para definir la cinemática este mecanismo articulado, cuyo tamaño y movimientos de cierre son los más similares a los de un dedo humano. Para este estudio se han utilizado las características del dedo índice.

TABLA 1

ARTiCULACIONES Y LíMites de La MaNo humana [8].

\begin{tabular}{l|l|l|l|l}
\hline Parte & Juntura & DOF & $\begin{array}{l}\text { Ángulo } \\
\text { Flexión } \\
\text { Extensión }\end{array}$ & $\begin{array}{l}\text { Ángulo } \\
\text { Abducción } \\
\text { Aducción }\end{array}$ \\
\hline Dedos & DIP & 1 & 60 & - \\
Dedos & PIP & 1 & 100 & - \\
Dedos“ & MCP & 2 & 90 & 60 \\
Pulgar & IP & 1 & 85 & - \\
Dedos & MCP & 2 & 50 & 30 \\
Dedos & CMC & 2 & 120 & 45 \\
\hline
\end{tabular}

Para entender cómo funciona el mecanismo, se hace a continuación la clasificación de éste en grupos de Assur (ver figura 4):

$I D_{1,0^{-}}>I I G G G_{2,3^{-}}>I I G G G_{4,5^{-}}>I I G G G_{6,7^{-}}$ $>I I G G G_{8,9}{ }^{->I I G G G_{I 0, I I}}$

El actuador de tipo rotacional es acoplado a un tornillo sin fin el cual tiene un tornillo para así obtener un movimiento de tipo lineal, por la forma como están acopladas las falanges el movimiento general se hace a través del sistema de barras.

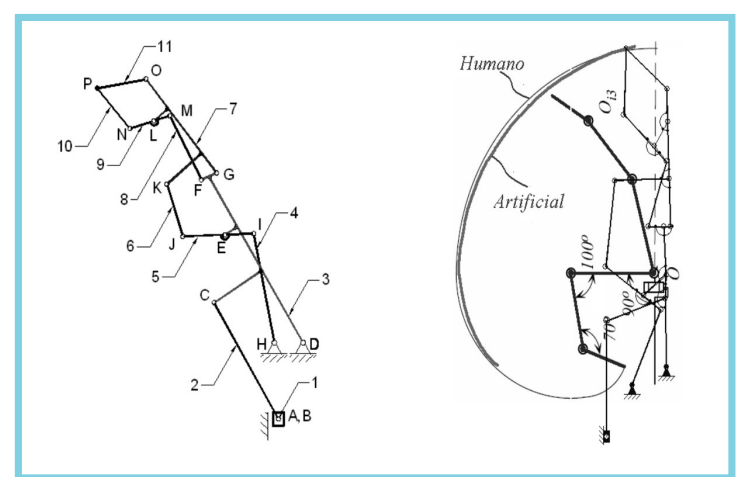

Figura 4. Clasificación de los grupos estructurales y espacio de trabajo.
El actuador utilizado es de tipo rotacional, y se encuentra acoplado a un tornillo sin fin, permitiendo así la realización de un movimiento de tipo lineal, y por otro lado el movimiento general del dedo; como se puede observar en la figura 5 , es realizado a través de un mecanismo de barras que está inserto dentro de las falanges.

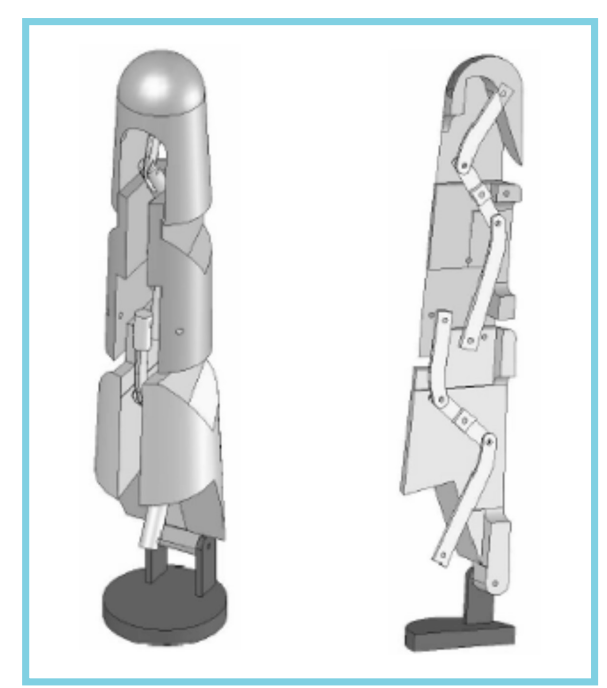

Figura. 5. Vistas de dedo diseñado donde se muestra como están conectados el sistema de barras con las falanges. 
Simulaciones en SolidWorks ${ }^{\circledR}$ y Visual Nastran ${ }^{\circledR}$, han sido desarrolladas para comprobar que el movimiento de cierre sea apropiado y que no hay interferencia mecánica, se muestra el prototipo construido en el laboratorio de procesos de mecanizado de la Universidad Tecnológica de Pereira con la característica de que el mecanismo es un mecanismo de barras y está dentro de los eslabones como muestra la figura 6 .

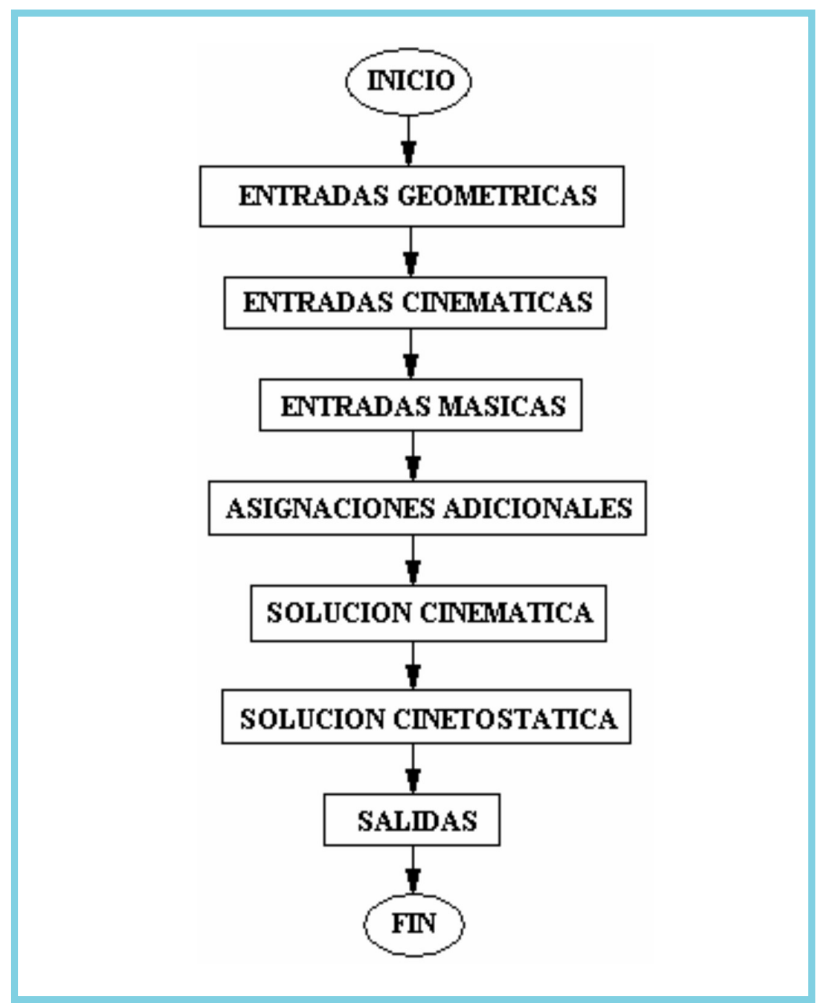

Figura 6. Algoritmo para estudiar el mecanismo.

La tabla 2 lista las analogías tenidas en cuenta entre el sistema biológico, dedo índice humano y el dedo mecánico.

\section{TABLA 2}

ELEMENTOS DEL DEDO DESARROLLADO

\begin{tabular}{l|l}
\hline Sistema biológico & \multicolumn{1}{c}{ Dedo mecánico } \\
\hline Huesos & $\begin{array}{l}\text { Resina, compuestos } \\
\text { plásticos } \\
\text { Revolución } \\
\text { Sistema palancas en acero } \\
\text { Articulaciones } \\
\text { Tendones } \\
\text { Músculos }\end{array}$ \\
\hline
\end{tabular}

\section{Pruebas y resultados}

Se realizó el análisis cinetostático de mecanismo del dedo robot. Para ello se recurre al análisis y síntesis de mecanismos por grupos de Assur. El análisis se inicia con la solución gráfica de las posiciones obtenidas con el mecanismo en un software CAD, se calcula manualmente las fuerzas y reacciones que se generan en cada junta y posteriormente se implementa un programa de computador sobre la plataforma de Matlab V7.0 para la solución de las ecuaciones, luego se comparan los resultados obtenidos en forma gráfica y en forma manual, así se obtuvo que el error es mínimo (en torno de $0,006 \%$ ) lo que implica que las soluciones fueron aceptables.

Pruebas experimentales han sido desarrolladas con el prototipo. Para estas experiencias se ha utilizado un instrumento virtual desarrollado sobre LabView® para la generación de una señal de PWM ${ }^{1}$ para control de velocidad y sentido de giro del actuador, además del instrumento virtual para la medición de fuerzas las cuales son registradas en los sensores (tipo Flexiforce ${ }^{2}$ ) durante una prueba de contacto.

Una secuencia de movimiento del dedo es mostrada en la figura 7, donde son presentadas tres posiciones: superior, intermedia e inferior. En la figura 8 son presentados resultados de simulación.

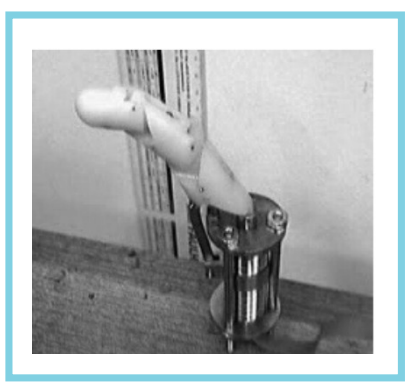

(a)

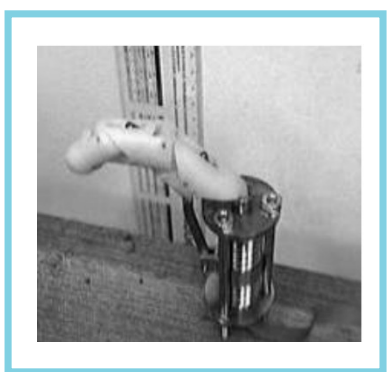

(b)
[Angl] Pulse width modulation: modulador de ancho de pulso. $\underline{\text { www.tekscan.com/flexiforce.html }}$ 


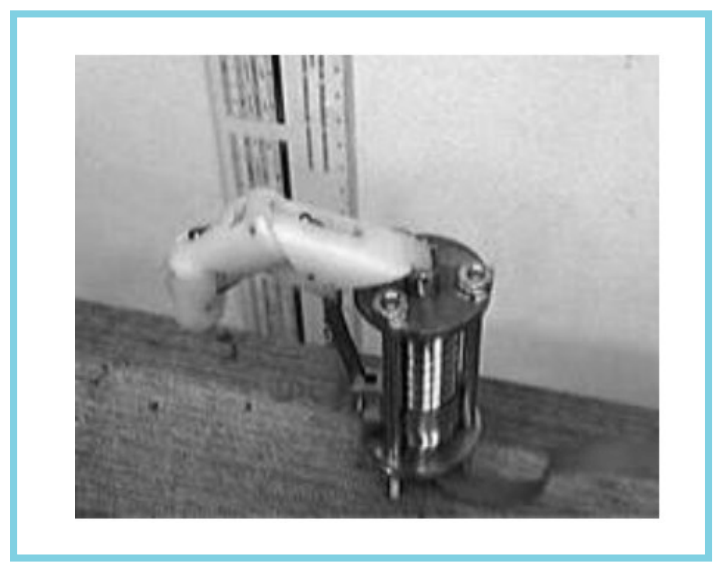

(c)

Figura 7. Dedo mecánico en las posiciones: (a) superior, (b) intermediaria, (c) inferior.

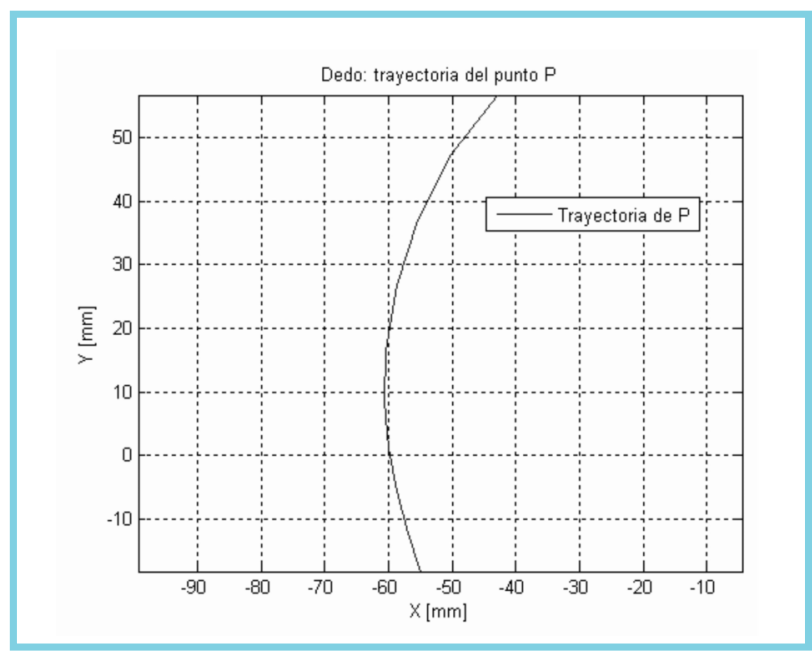

(a) Trayectoria del punto $\mathrm{P}$

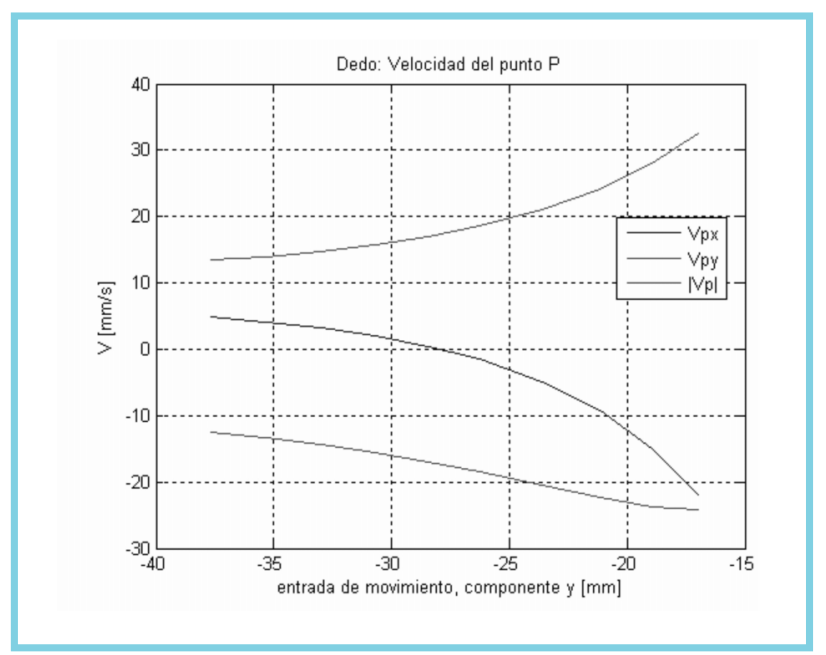

(b) Velocidad del punto $\mathrm{P}$

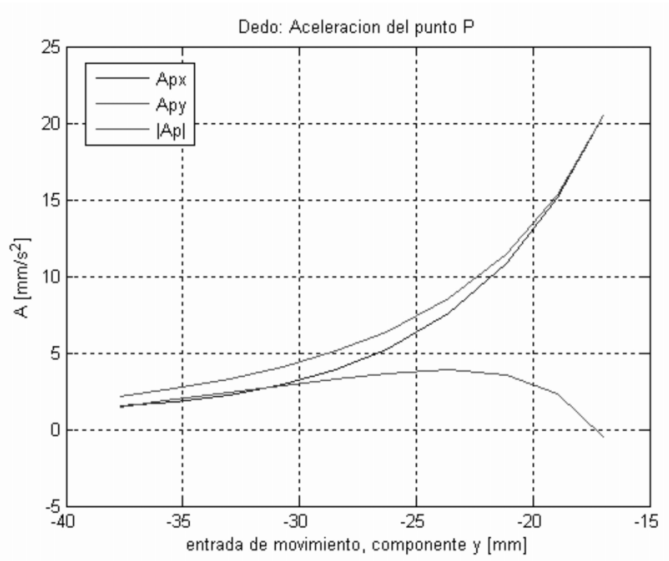

(c) Aceleración del punto $\mathrm{P}$

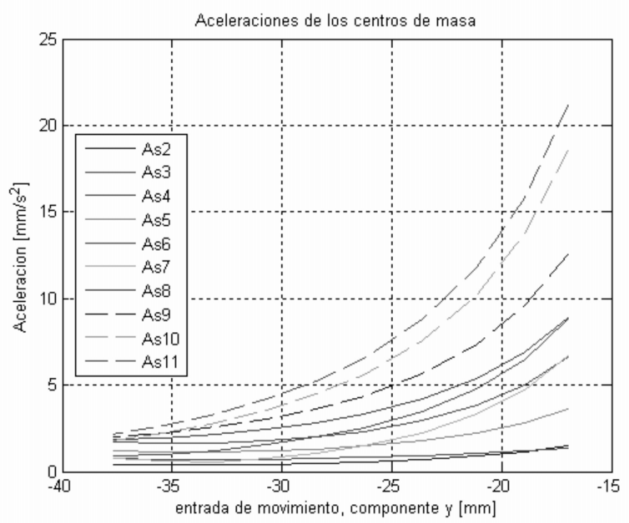

(d) Aceleraciones de los centros de masa

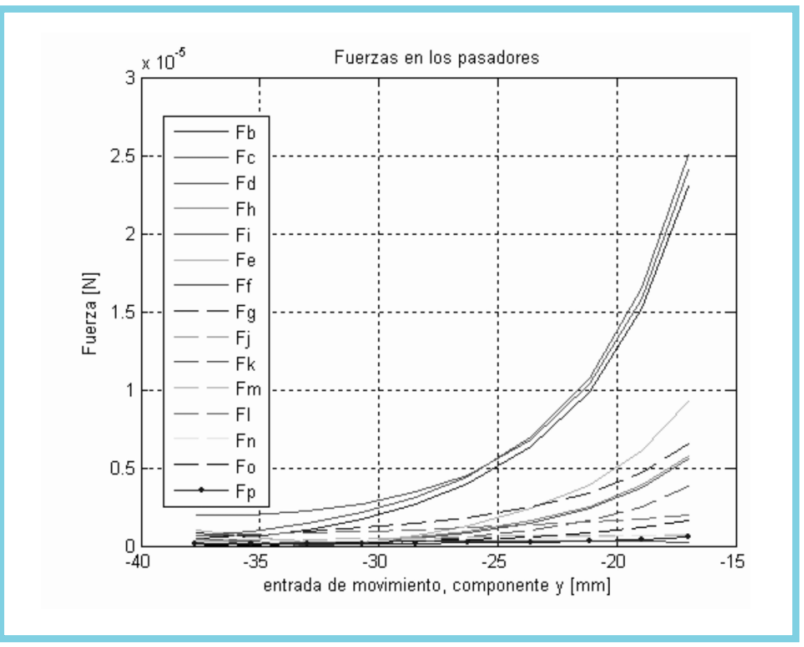

(e) Fuerzas en los pasadores 


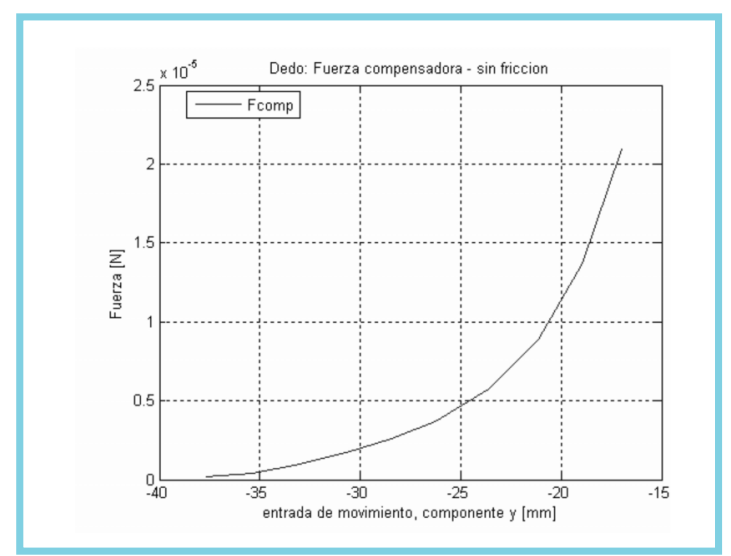

(f) Fuerza compensadora - sin fricción

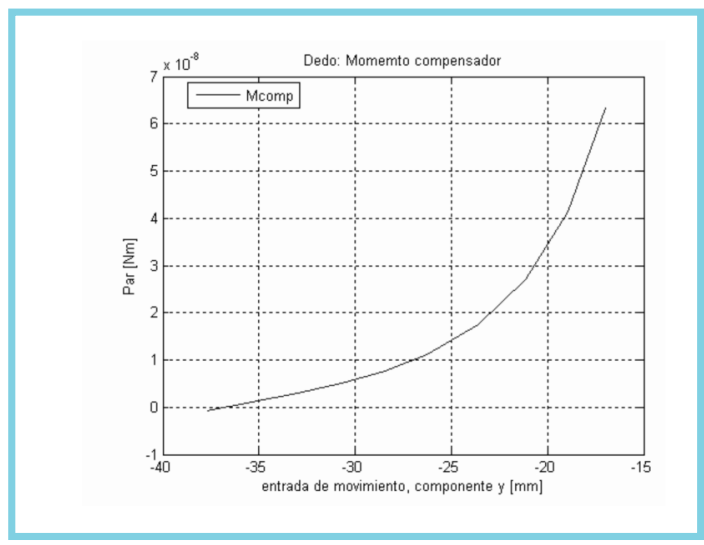

(g) Momento compensador

Figura 8. Resultados de las simulaciones obtenidas con Maltab®.

Los resultados obtenidos a través del software Matlab® confirman la suposición de que las fuerzas son pequeñas comparadas con los cálculos estáticos realizados en forma manual para las diferentes posiciones del mecanismo. Para una rosca $A C M E$ se calcula la fuerza compensadora y a partir de esta fuerza son calculadas las otras fuerzas para cada uno de los pontos de la trayectoria.

En la tabla 3 son presentados los principales resultados obtenidos con ayuda de Matlab®, donde se puede verificar que la trayectoria de un determinado punto $P$, en el extremo del mecanismo, describe unas posiciones aceptables en relación al movimiento de un dedo humano, como por ejemplo, la velocidad que se obtuvo en forma experimental fue de $5 \mathrm{~mm} / \mathrm{s}$, y el resultado obtenido en simulación es de $4,95 \mathrm{~mm} / \mathrm{s}$.

Otra técnica para la simulación de mecanismo utilizada es por medio del toolbox de Matlab® Simmechanics, la figura 9 muestra la referencia tenida en cuenta para el montaje. En la figura 10 se presenta la respectiva implementación.

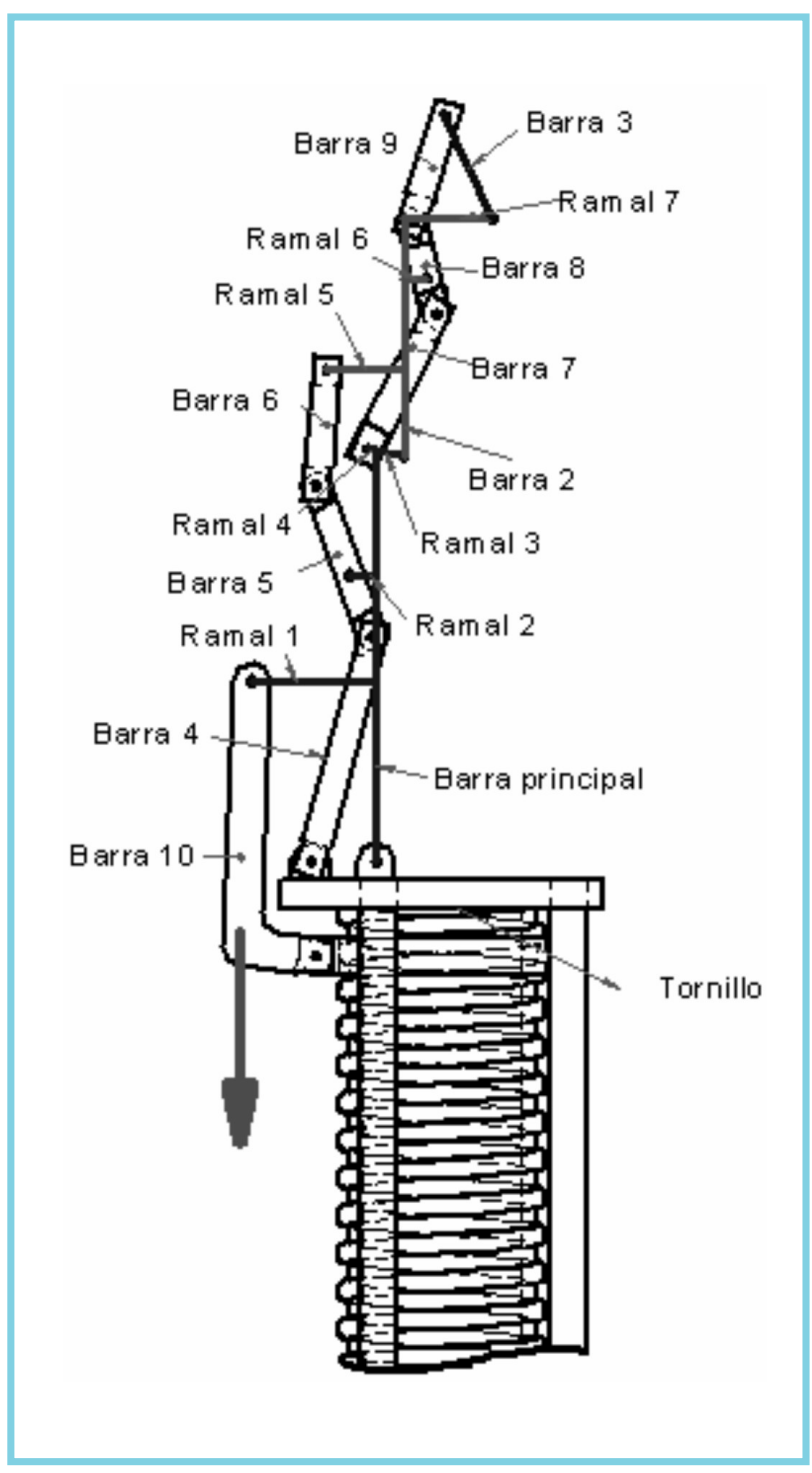

Figura 9. Referencia para el montaje en Simmechanics. 


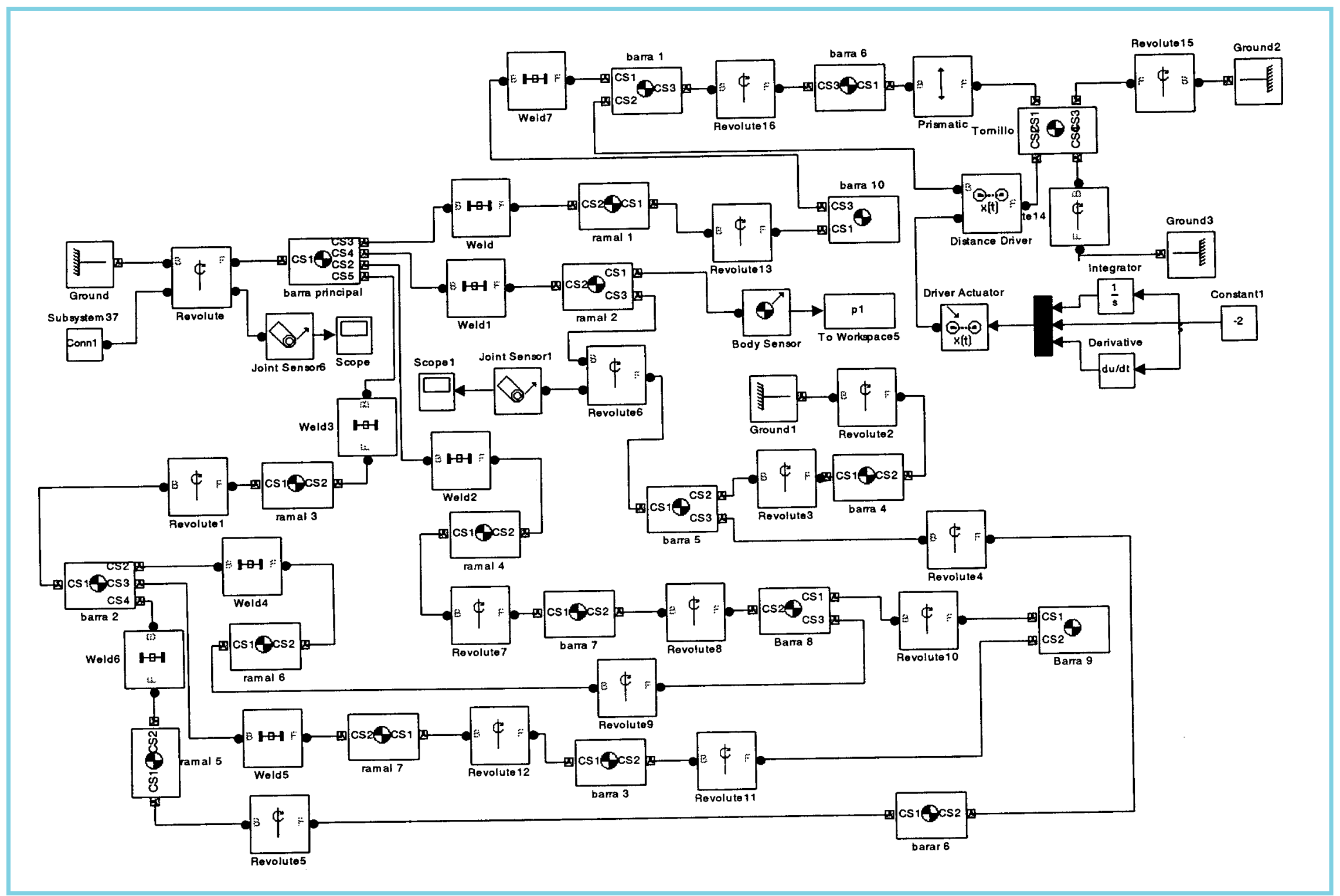

Figura 10. Implementación del mecanismo en Simmechanics de Matlab® 


\section{Conclusiones}

A través de este trabajo se plantearon las estrategias para la implementación de un dedo robótico en el desarrollo de grippers para aplicaciones industriales o fines protésicos. La mayoría de los desafíos de la fabricación del dedo robot se presentaron debido a su forma de características antropomórficas. Esto hace difícil la manufactura de ciertas piezas, por ejemplo, las barras de transmisión y los eslabones que conforman las falanges, pues éstos deben cumplir con unas dimensiones particulares.

Otro hecho importante es lograr que el dedo robot tenga otro grado de libertad en su base para lograr mayor destreza en la manipulación de objetos de geometrías complejas, y a la vez calcular las fuerzas que se generan en la nueva configuración.

El hecho de que el robot fue construido en forma manual significó demoras en la construcción final, debido a los errores humanos inherentes en este tipo de trabajos. Pero también hay que resaltar que las formas logradas satisfacen las características antropométricas (forma, tamaño), variables a tener en cuenta. Ahora bien, es claro que las técnicas modernas para el maquinado de piezas complejas pueden facilitar y minimizar muchos errores, por ejemplo, empleando técnicas de CAD/CAM/ CAE o estereolitografía,

El material utilizado (Teflón) para la fabricación de las falanges, tiene características mecánicas aceptables, además de ser de bajo costo, lo que hace que sea tenido en cuenta en futuros diseños de bioingeniería.

Este dedo robot facilitará la implementación de una mano completa, la cual puede ser usada en aplicaciones de inteligencia artificial con robots humanoides, obviamente incorporando algunas mejoras para dotar de sensores para la adquisición de diferentes sensaciones, como por ejemplo temperatura, para control dinámico del sistema en lazo cerrado.
Un sensor, en detalle, se podría aplicar a todos los elementos constitutivos del dedo robot, por ejemplo, una uña podría ser utilizada, como sus contrapartes biológicas; esta uña sería un transductor importante de la información con el cual el dedo robot podría raspar, cavar, y golpear ligeramente objetos, una propuesta se muestra en la figura 11 .

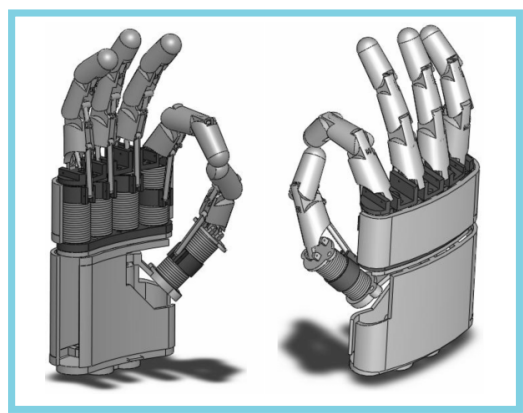

a) Mano robótica

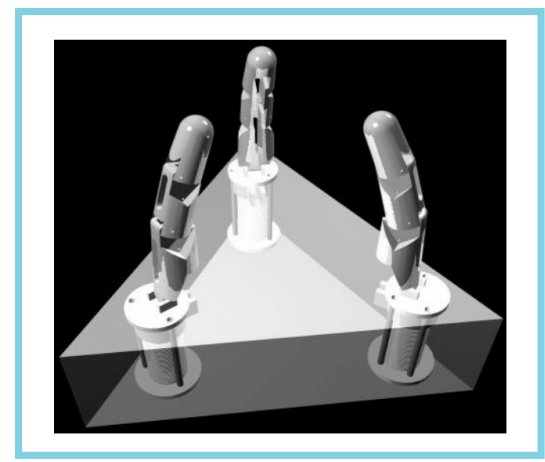

b) Gripper de tres dedos

Figura 11. Construcción futura usando el dedo como módulo.

Este tipo de trabajos requieren del uso de las diferentes teorías para el análisis y síntesis de mecanismos, y demuestra que la ingeniería puede tomar como fuente de inspiración la biomecánica para el desarrollo de nuevos sistemas que emulen el movimiento humano.

Recibido: Mayo de 2007

Aceptado: Octubre de 2007 


\section{Referencias}

1.Matthew, T., Mason, J. Kenneth Salisbury Jr. "Robot hands and the mechanics of manipulation". The MIT Press Series in Artificial Intelligence. 1985.

2. Jacobsen, S. E. Wood, J. E. Knutti, and D. F. Biggers, K "The UTAH/MIT dextrous hand: work in progress". In Robot, Grippers, Springer-Verlag, Berlin. 1986.

3. Nakano, M. Fujie, M. and Hosada, Y."Hitachi's robot hand". Robotics Age, julio 1984, 6 (7).

4. Butterfass, J. Hirzinger, G. Knoch, S. and Liu. H. "DLR's multisensory articulated hand" In Proc of the 1998 IEEE Int. Conf. on Robotics and Automation, Leuven, Bélgica, mayo 1998.

5.Laschi, C., Dario, P. "Grasping and manipulation in humanoid robotics", Scuola Superiore Sant Anna, Italia.

6.Lovchik, C.S. and Diftler, M.A.. "The robonaut hand: A dextrous robot hand for space". In Proc of the 1999 IEEE Int. Conf. on Robotics and Automation, Michigan, mayo 1999.

7.Schulz, S., Pylatiuk, C. "A new ultra light anthropomorphic hand". Inst. of Applied Computer Science Research Center of Karlsruhe, Alemania.
8.Taylor, G.L.,s Schwartz R.J. "The anatomy and mechanics of the human hand", Artificial Limbs, 1955; 2: 22-35.

9.An, K.N., Chao, E.Y., Cooney, W.P. and Linscheid, R.L. "Normative model of human hand for biomechanical Analisys". Journal of Biomechanics, 1979; 12: 775-788.

10.Cunha. F. Dynnikov, V "Detalhes construtivos de uma protese antropomorfica para membros superiores - um estudo das transmisões e acionadores", XV Congreso Brasileiro de Engenharia Mecanica, 1999, Sao Paulo. Disponible en: http://www.sel.eesc.sc.usp.br/ labciber/fcunha/COBEM99.pdf

11.Dario, P., Lashi, C., Chiara-Carroza, M. “A Human-like Robotic manipulation System Implementing Human Models of SensoryMotor Coordination", disponible en: http:// www-arts.sssup.it/download/papers/

12.Ocampo, G.A., Rondón, J. "Diseño y construcción de una mano robot de cuatro dedos que imite los modelos prensiles humanos", Universidad Militar Nueva Granada, 2004.

13. Avilés, O., Simanca, P. "Diseño y construcción de un dedo para grippers robóticos", tesis de maestría, Facultad de Ingeniería Mecánica, Universidad Tecnológica de Pereira, 2006. 